\title{
Sexual dimorphism in immune function: The role of sex steroid hormones
}

\author{
Anna Mihailova ${ }^{1, *}$ and Indrikis Krams ${ }^{1,2}$ \\ ${ }^{1}$ Daugavpils University, Latvia \\ ${ }^{2}$ University of Latvia, Latvia
}

\begin{abstract}
There is evidence of the relation of sex steroid hormones and sexual dimorphism in immune system response to infectious diseases. The aim of this review was to identify the role of sex hormones in immune function and sexual dimorphism of immune reactions. Gonadal hormones together with the immune system play an important role in process of immune responses to the disease [1]. Estrogens, progesterone and testosterone have different impacts on immune cells and different gonadal hormones are of high importance for responses of innate and adaptive immunity [1,2]. Estrogens mainly enhance immune function while testosterone has a suppressive role. Higher progesterone during pregnancy leads to autoimmune disease remission and an elevated susceptibility toward certain infectious diseases [2-4]. The intensity and prevalence of viral infections are typically higher in males, whereas disease outcome could be worse for females [5]. Sexual dimorphism of immune function is based on different concentrations of sex hormones in males and females and on a specific mediating role of these hormones in immune function and response along with differences in innate and adaptive immunity.
\end{abstract}

Key words: sexual dimorphism, sex steroid hormones, immune system, infectious diseases.

\section{Introduction}

Sex is a biological variable that affects immune responses to both self and foreign antigens [6]. The authors specifically point to the difference in terms of "sex" and "gender", regarding the aim of identifying exact biological factors that influence the immune response and differences of those responses in males and females. Gender is the fundamental dimension, which people use to categorize themselves and others [7] and includes behaviour and activities that are determined by society or culture in humans [6, 8]. Focusing on sex as a factor that contributes to physiological and anatomical differences which influence exposure, recognition, clearance, and even transmission of micro-organisms [6], it is possible to find reasons for sexual dimorphism in susceptibility to diseases, physiological processes, occurring during fighting the infectious disease, and immune response to vaccination. This is because of evidence of relation of sex steroid hormones and immune system response to infection diseases [1-5]. Moreover, there is sexual dimorphism in immune system response. The aim of this review was to identify the role of sex hormones in immune function and sexual dimorphism of immune reactions.

\footnotetext{
* Corresponding author: anna.mihailova@du.lv
} 


\section{Material and methods}

Published literature was retrieved through searches of PubMed, SCOPUS and EBSCOHost MEDLINE in January and November 2016 with the use of appropriate controlled vocabulary (e.g., immune system, sexual dimorphism, and sex steroid hormones) and key words (e.g., immune system AND sex hormones, immune response AND sex hormones, immune system AND sexual dimorphism, vaccination AND sex hormones). Abstracts and articles with full texts were selected for the review fitting the criteria of inclusion by selected theme and content.

Division of information selected was done according to two major themes: infectious diseases and autoimmune diseases as shown in Fig. 2. Based on this information, stratification of the review was formed as follows:

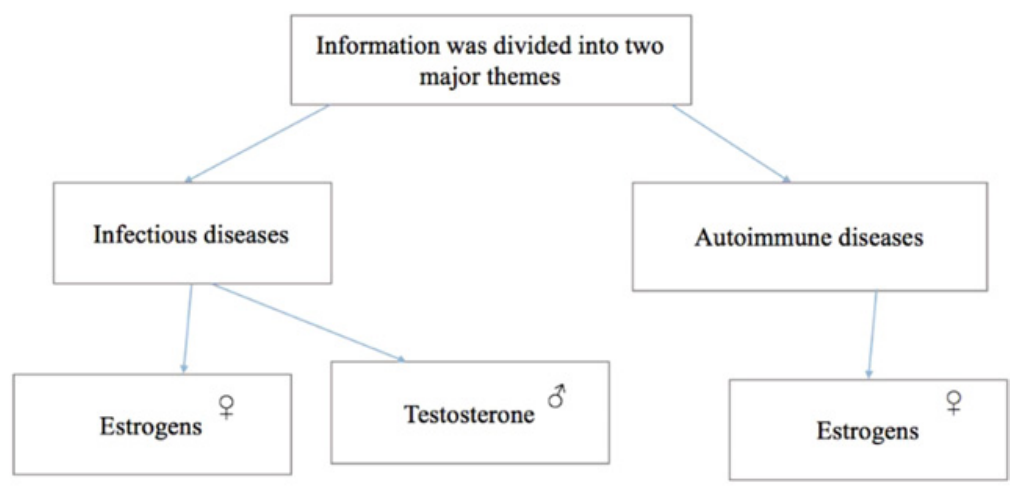

Fig. 1. Stratification of articles according to the major themes (sex difference indicated in symbols where $q$ stands for females, $o^{7}$ for males).

\section{Sex hormones and immune function}

Sex is defined by the differential organization of chromosomes, reproductive organs, and sex steroid levels [6]. As the differences are present biologically according to genetics and anatomical structures, as well as physiological factors such as hormones, it is clear that the differences in functions in different sexes must be obvious, specifically in the function of immune system. Sex steroids of reproductive system are one of the major factors that regulate the immune system due to the presence of hormone receptors on the membranes of immune cells [9]. The interaction of sex hormones and immune cells through the receptors on these cells effects the release of cytokines which determines the proliferation, differentiation, and maturation of different types of immunocytes and as a result the outcome of inflammatory or autoimmune diseases [9]. The interaction of two major systems of the human body, the immune system and the endocrine system, can be illustrated as in Fig. 2.

\subsection{Sexually dimorphic susceptibility to infectious diseases}

Zuk and Stoehr (2010) in their review article [10] pointed to the fact that males are more likely to suffer from parasites and that incidence and pathogenesis of parasite infections are more profound in males than in females [3]. In humans, the intensity and prevalence of viral infections were found to be higher in males [5]. 


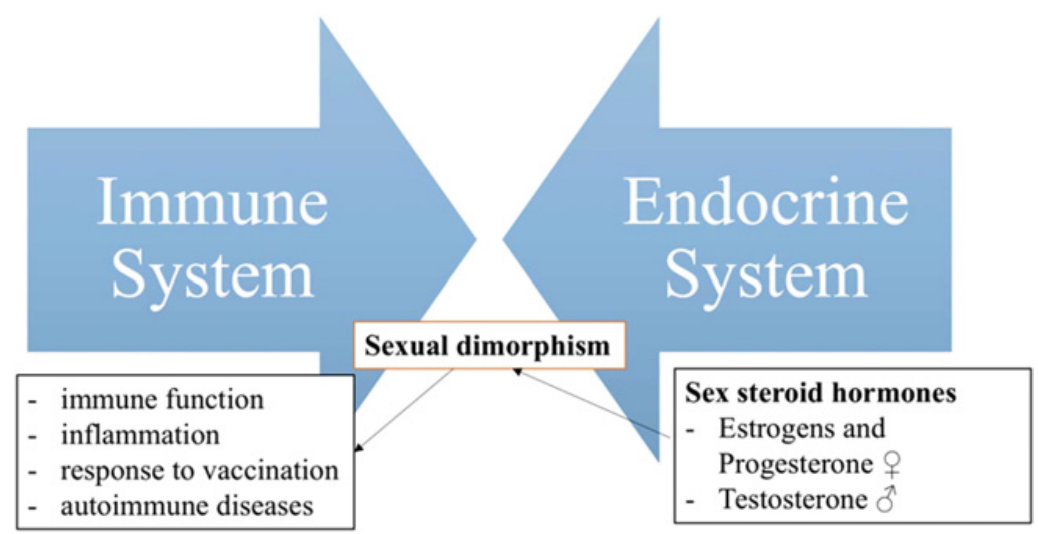

Fig. 2. Scheme of connection of immune and endocrine systems as the basis of sexual dimorphism (sex difference indicated in symbols, there $q-$ female, $\sigma^{7}-$ male).

Men are more susceptible to many infections caused by viruses, bacteria, parasites, and fungi. As summarized in a review by Giefing-Kröll, Berger, Lepperdinger, and GrubeckLoebenstein (2015), men are more predisposed especially to environmental- and vector-borne diseases such as leptospirosis, schistosomiasis, brucellosis, rabies [11, 12], leishmaniasis, pulmonary tuberculosis, hepatitis A [12], meningococcal or pneumococcal infections [2].

The major contributors of sexual dimorphism in susceptibility to infectious diseases appear to be:

1) lifestyle as a gender-specific factor, for example, smoking, alcohol, risk-taking, medical therapies $[13,14]$, exposure to microorganisms, access to healthcare or health seeking behaviour that affects the course of infection [6];

2) biological determinants of sexual dimorphism as sex-specific factors, for example, $X$ chromosome diploidy, differences in immune and hormonal responses $[14,15]$.

\subsection{Sex steroid hormones as modulating factors of immune response}

Recent studies not only try to describe sex differences of susceptibility to infection diseases and parasites, but also answer the question on reasons for those differences. In terms of the endocrine system, the prevailing hypothesis for immunological differences between the sexes is that sex steroids, particularly testosterone (T), estradiol (E2), and progesterone (P4), influence the functioning of immune cells [5]. Observational studies showed that sexrelated differences in the immune responses are modulated by sex hormones, particularly testosterone and estrogen [3]. Gonadal hormones together with the immune system play an important role in the process of immune responses to the disease [1]. Research groups of Lourenço et al. (2008) and Giefing-Kröll et al. (2015) in their reviews pointed at different influences of estrogens, progesterone and testosterone on immune cells. Gonadal hormones have specific effects on the male and female immune function at both cellular and molecular levels [2]. Lourenço et al. (2008) acknowledged that women show more effective and potentially protective humoral and cell-mediated immune responses that cause an immune advantage compared with males [1]. As the immune system comprises two major pathways, innate and adaptive immunity, different gonadal hormones take a leading role in these two defense levels. Sex differences in immune responses have evolved in organisms ranging from insects to lizards, birds and mammals; in all of these species, both innate and adaptive immune responses are typically lower in males than in females [6]. 


\subsubsection{Role of estrogens and progesterone in infectious disease pathogenesis}

According to Fish (2008), estrogen receptors are expressed in most cells of the innate and adaptive immune system. This includes T cells, B cells, neutrophils, macrophages, dendritic cells (DC), and natural killer (NK) cells [16]. Klein (2012) suggested that induction of genes associated with toll-like receptor (TLR) pathways and antiviral type I interferon responses are higher in females, and that number and activity of innate immune cells, including monocytes, macrophages, and DC as well as inflammatory immune responses in general are higher in females than males [5].

Estrogens are mainly enhancing immune function and immune response, while testosterone has a suppressive role. Higher progesterone during pregnancy often leads to autoimmune disease remission, as well as to an elevated susceptibility towards certain infectious diseases $[1,2,4]$.

Robinson et al. (2011) found that elevated $17 \beta$-estradiol (E2) protects females from influenza A virus pathogenesis by suppressing inflammatory responses. Protective effects of estradiol on proinflammatory cytokines and chemokines, morbidity and mortality were primarily mediated by signaling through estrogen receptor $\alpha$. Moreover, women suffer a worse outcome from influenza A virus infection than men, but estradiol helps to prevent it [17].

Hayashida et al. (2010) investigated the role of $17 \beta$-estradiol (E2) in immune response to hepatitis $\mathrm{C}(\mathrm{HCV})$. They based their research on the fact that the male gender is one of the critical factors in progression of hepatic fibrosis due to chronic HCV infection; thus, female hormones may play a role in delaying the progression of hepatic fibrosis [18]. The results showed that $\mathrm{E} 2$ has a positive effect on suppressing production of $\mathrm{HCV}$ infectious particles (virions) and thus enhances the immune function against the viral infection.

Males and females differ in their responses to infections caused by many viral pathogens, including human immunodeficiency virus (HIV) [19]. Supporting the sexual dimorphism theory in immune-competence, Klein (2012) referred to previously published studies made by Napravnik, Poole, Thomas and Eron Jr. (2002) and state that females have approximately $41 \%$ less HIV loads, and females have higher antiviral responses to HIV than males. In males, HIV infection is mainly associated with lowering of androgen hormones. However, apart from the reduced androgen hormones level, in men HIV infection increases estrone and E2 concentrations [5].

Rodriguez-Garcia et al. (2013) tried to identify the direct effect of E2 and ethinyl estradiol (EE) in HIV-infection in women [20]. Their findings indicate that E2 has a direct effect on susceptibility of HIV-target cells to infection and that E2-treatment reduces viral entry $2 \mathrm{~h}$ after challenge and increase MIP-1 $\beta$ secretion [20]. So they proved that E2 has an inhibitory effect on target cell infection and this involves cell-entry related mechanisms. This also proves the role of E2 in pathogenesis of HIV infection and the immune enhancing role of the hormone.

In terms of effects of progesterone, Klein (2012) showed that P4 can modulate the function of plasmacytoid DCs (pDCs), and women with higher level of P4 in plasma have greater numbers of IFN-a producing pDCs in response to the HIV TLR7 lig and than women with lower P4 concentrations [5]. Additional findings indicate the same immunosuppressive role of higher $\mathrm{P} 4$ concentration during P4-based hormone contraceptive use, which is expressed in higher activity of HIV-1, loss of CD4+ T cells and progression of the disease [5].

According to findings in studies in animals, gonadal hormones may have various effects on course of bacterial infection. As it has been revealed in research of Rank et al. (1982): "Treatment with estradiol was found to markedly influence the course of genital infection 
with the chlamydial agent of guinea pig inclusion conjunctivitis, producing infections of greater intensity and longer duration than those in control animals. Moreover, pathogenesis was altered in that ascending infection was observed, resulting in endometritis, cystic salpingitis, and cystitis [21]". More recent research on human subjects done by Agrawal et al. (2008) studied the role of cervical dendritic cell (cDC) subsets, co-inflammatory molecules, cytokine secretion profile and E2 in development of further complications to Chlamydia trachomatis infection. The results suggest that a differential activation of subsets of DC, cytokine secretion pattern and hormonal levels can be responsible for modulating the immune response to chlamydial infection [22]. Thus, a higher level of E2 in women enhances the pathogenic activity of Chlamydia directly by helping in its intracellular development or indirectly by modulation of cytokine secretion profile of immune cells [22]. Moreover, the same findings were in previous studies in humans, indicating that women are more susceptible to chlamydial infection under $\beta$-estradiol influence, and this is due to more chlamydial organisms that are isolated during the proliferative part of the cycle [23]. Thus, unlike with influenza A, E2 has a negative effect on immune system response to Chlamydial infection and enhances inflammation. Overall, the conclusion can be made that E2 does not help to fight the bacterial infection compared with the previous examples of viral infections.

\subsubsection{Role of estrogens and progesterone in autoimmune disease pathogenesis}

Supporting the opinion that immune system shows a major sexual dimorphism in vertebrates, Dumont-Lagacé, St-Pierre and Perreault (2015) reported that relative to males, females usually display stronger immune response to vaccination and infection but suffer a higher propensity to many autoimmune diseases [24]. This was stated earlier by other authors [1,25-27], pointing at higher incidence of an array of autoimmune disorders in women. What is the role of estrogens in this process? Does E2 lower the risk of autoimmune diseases in women? Some reports suggested that systemic lupus erythematosus (SLE) patients experience an increase in flares during pregnancy, possibly due to the sustained increased levels of estrogen [28]. There is evidence from Lahita and Bradlow (1981) research, what Li et al. (2009) reported, that in patients with SLE and their first-degree relatives had elevated level of E2 [29]. SLE precipitated or exacerbated after the commencement of oral contraceptive use [28], which increases the level of E2. Li et al. (2009) have carried out a study on influence of $17 \beta$-estradiol on $\mathrm{pDC}$ in response to $\mathrm{CpG}$ in mice. Their findings indicate that $\mathrm{E} 2$ could exacerbate pDCs' activation with $\mathrm{CpG}$, which further activates B cells to regulate susceptibility to auto-antigens [28]. This suggests that scientists and physicians have suspected that steroid hormones such as estrogen may be key regulators of autoimmune diseases including SLE [28], and females have higher prevalence to autoimmune diseases compared with men.

Dumont-Lagacé, St-Pierre and Perreault (2015) found that sexual dimorphism in thymic epithelial cell (TEC) was particularly conspicuous where it was mainly regulated by levels of testicular androgens [24]. It was shown that TEC proliferation and thymic output were higher in females. In males, numerous genes that are instrumental in thymocite development were repressed in male cTECs. However, the number of these genes was expressed more in males compared with females. The possible reason for this finding is the enhanced cell survival coupled to impairment of cell differentiation in males. What is the reason of the superior proliferative potential in cTEC in females? The answer is the pregnancy-induced cTEC renewal potential, the process, which is progesterone-dependent [24]. Klein and Flanagan (2016) suggested the anti-inflammatory effect of progesterone to be similar to that of E2. They also mentioned that progesterone receptors could be found in many different immune cell types (NK cells, macrophages, DCs, and T cells) [6]. 


\subsubsection{Role of testosterone in infectious disease pathogenesis}

According to the paper of Morton, Weyant, Siegel and Golding (1981), modulating role of sex hormones and sexual dimorphism were studied since the end of the $20^{\text {th }}$ century, and researchers proved that women have had fewer infections, higher baseline IgM levels, and better humoral responses to antigens [30]. Thus, the question was whether sex, testosterone administration and castration have any effect on immune responses. Previous findings showed that testosterone administration can ameliorate the autoimmune disease in female mice, but castration, in contrast, accelerates this disorder in male mice [31, 32]. Morton et al. (1981) did not prove this. It has been found that castration affects the regeneration and/or differentiation of the haemopoietic stem cells, and the authors suggested: "Male mice that were irradiated and castrated showed significantly higher anti-sheep red blood cell responses than noncastrated, irradiated mice, demonstrating that normal levels of endogenous sex steroids may also modify immune development in an expanding haemopoietic system" [33]. It was shown that mice responded to sheep red blood cells dependent on their testosterone levels while testosterone was present in mice of both sexes. Furthermore, testosterone had a negative impact on antibody responses that was observed by Morton et al. (1981). In the study of Lourenço et al. (2008), it was found that the increased prevalence of susceptibility to parasites in males is linked with the immunosuppressive effect of testosterone. This male sex hormone also affects the immune response of Calomys callosus to Trypanosoma cruzi infection, they also reported that in other studies on mice susceptibility to infection was also testosterone dependent [1].

Triphati and Singh (2014) support the above evidence summarising findings of several studies and state that "use of sex steroids or its receptor-antagonists or gonadectomy in animal models for studying the patho-physiology and immune-endocrinology has provided direct evidence for the role of sex steroids in immune modulation" [34]. Lourenço et al. (2008) state that the use of $10 \mathrm{mg}$ testosterone implants was the factor that increased susceptibility of female mice to Strongyloides ratii and female mice showed similar recovery from the infection as found in males [1]. In addition, in female mice, as stated above, estrogens can influence resistance to parasitic diseases [1, 27, 35], which may enhance (in males) or suppress (in females) susceptibility to parasite infections.

Mills et al. (2009) carried outastudyon voles and investigated trade-offs between immune system and testosterone [36]. Males from a low-immune group had higher plasma testosterone levels than those from a high immune group. Significantly higher testosterone levels in males from the low-immune group continued into the breeding season. In addition, a significant negative correlation between $\mathrm{T}$ and $\log 10 \mathrm{IgG}$ level and a nonsignificant trend for a negative correlation between $\mathrm{T}$ and $\log 10$ anti-BGG antibody response were found, further confirming an immune response testosterone genetic trade-off [36]. These findings support the role of testosterone in the trade-off between immune function and sex hormones, and specifically immunosuppressive role of testosterone.

Different studies on animals, such as fresh water snakes [34], the red-winged blackbird (Agelaius phoeniceus) [37], the house sparrow (Passer domesticus) [38], the Siberian hamster (Phodopus sungorus) [39], point to controversial effects of testosterone on immune function. Triphati and Singh studied the effects of in vitro testosterone on phagocytosis and cytotoxic responses of splenic macrophage as well as on mitogen induced splenic lymphocyte proliferation in fresh water snake males. They found that in vitro testosterone treatment significantly decreased the percentage phagocytosis by splenic macrophages and further it was significantly correlated with the in vitro testosterone concentration [34]. The authors studied the differential role of testosterone concentration on Nitroblue Tetrazolium salt (NBT) reduction. They found that NBT reduction was decreased at $10 \mathrm{ng} \mathrm{ml}^{-1}$, but was 
unaffected at $100 \mathrm{ng} \mathrm{ml}^{-1}$, and again decreased at $1000 \mathrm{ng} \mathrm{ml}^{-1}$, and the decrease was the greatest at $1000 \mathrm{ng} \mathrm{ml}^{-1}$ testosterone concentration. Nitrite release was significantly reduced by in vitro testosterone treatment in a concentration dependent manner [34], and a higher nitrite release was observed in lower testosterone levels. These results suggest that lower testosterone levels suppress immune system function in reptiles, and reveal the differential role of testosterone concentration, pointing out a non-linear relationship: the marginal (lowest and highest) testosterone concentrations both lower NBT concentration. The authors explain such a biphasic effect by the process of production of an increased amount of $\mathrm{O}_{2}^{-}$in reptilian splenic macrophages and that testosterone has a modulating role on the metabolic pathway in this process.

The findings by Merrill et al. (2014), Greenman, Martin and Hau (2005) in birds and Mills et al. (2009) in voles showed no direct relation of testosterone to immune system function and testosterone was not the primary factor to suppress the immune function [36-38]. Merrill et al. (2014) found that testosterone was not related to any parasite parameter, and among all males, testosterone was not correlated with bacteria-killing ability (BKA). However, after adjusting for age, a significant negative relationship between testosterone and BKA was observed in older males. Adult males had significantly higher levels of testosterone and adults with higher testosterone levels had lower BKA than-younger males [37]. The authors suggested a possible trade-off between the effects of immune system and gonadal hormones. "However, some studies documented negative effects of testosterone on measures of immunity (e.g., Duffy et al., 2000; Peters, 2000; Casto et al., 2001; Buchanan et al., 2003), while others have found no link (Ros et al., 1997; Hasselquist et al., 1999; Nunn et al., 2009) or positive effects/associations (Evans et al., 2000; Roberts and Peters, 2009; Ezenwa et al., 2012)" [37]. Merrill et al. (2014) suggested that something else than testosterone may be directly driving the associations between semistatic signals and parasite levels, as well as immune function [37]. Taking into account that there was no significant negative correlation found in younger males, testosterone is not the direct factor affecting BKA.

In the study by Greenman, Martin and Hau (2005) the authors attempted to find out how do trade-offs between costly life-history states and immune activity take place in birds. The immune response of captive male house sparrows in photoperiodically induced states of reproduction, moult, and overwintering (i.e., non-breeding, no moulting) was observed. Their finding showed that passerine birds show seasonal modulation in immune function, even under benign environmental conditions. However, even though testosterone is often mentioned as a strong immunosuppressant, they found that it is not fully responsible for theobserved seasonal modulations. This is because mitogen phytohemagglutinin (PHA) induced wing web swelling was not reduced even when testosterone was present at high levels in the peripheral circulation [38]. This was observed in both breeding and nonbreeding birds. The researchers concluded that hormones might mediate trade-offs between the immune system and life history events but probably function in a state-dependent manner [38].

The results of Lourenço et al. (2008) demonstrate that Calomys callosus females demonstrated higher immune activity and higher Con A proliferation index than males after both were infected by Trypanosoma cruzi. The authors state that this is a sign of a better control of the infection mainly in the acute phase. However, the authors also suggest that during the late chronic phase, although parasitemia levels are undetectable, both sexes display an apparent normal profile with absence of amastigote burdens' [1]. In male subjects, research held on male Calomys callosus infected with the $\mathrm{Y}$ strain of Trypanosoma cruzi higher resistance with lower lysis indexes were observed after orchiectomy compared to intact and sham males [40], indicating the suppressive role of testosterone. 
Giefing-Kröll et al. (2015), making a review "How sex and age affect immune responses, susceptibility to infections, and response to vaccination" showed that "testosterone exerts immune suppressive effects thereby increasing the severity of malaria, leishmaniasis, amebiasis, and tuberculosis, while at the same time supporting the clearance of toxoplasmosis" [2]. Thus, the authors suggest a suppressive role of testosterone as a sex hormone on the immune system functioning in humans.

\section{Conclusions}

Sexual dimorphism of the immune function is based on different concentrations of sexual hormones in males and females and on a specific mediating role of these hormones in immune function and immune response along with differences in innate and adaptive pathways of those responses.

Women have higher humoral and cell-mediated immune responses and estrogens enhance innate and adaptive immune functions. Testosterone has a suppressive effect and immune responses are typically lower in men than in women. The intensity and prevalence of viral infections are typically higher in males, but disease outcome is worse for females. Females have higher susceptibility to some bacterial infections (e.g., Chlamydial infection). Women are more predisposed to autoimmune diseases due to the immune-enhancing role of estrogen. Specifically, high progesterone levels during pregnancy often lead to autoimmune disease remission and an elevated susceptibility toward certain infectious diseases.

We are thankful to Severi Luoto who kindly checked the English.

\section{References}

[1] A.M. Lourenço, A.M.A. Levy, L.C. Caetano, A.A. Carraro Abrahão, J.C. Jr., Res. Vet. Sci. 85 (2008)

[2] C. Giefing-Kröll, P. Berger, G. Lepperdinger, B. Grubeck-Loebenstein, Aging Cell. 14, $3(2015)$

[3] S.L. Klein, C. Roberts, Sex hormones and Immunity to Infection (Springer Verlag, 2010), pp. 1-2, 19-20

[4] Pazos et al. (2012), edited by S.L. Klein, C. Roberts, Sex and Gender Differences in Infection and Treatments for Infectious Diseases (Springer, 2015)

[5] S.L. Klein, BioEssays 34, 1050 (2012)

[6] S.L. Klein, K.L. Flanagan, Nat. Rev. Imm. 16 (2016)

[7] T. Meadow, Gend. Soc. 24(6), 814-837, edited by H.E. Owen, J. Halberstadt, E.W. Carr, P. Winkielman, PLoS One. 11(2), e0146328 (2016) doi: 10.1371/journal. pone. 0146328

[8] K.L. Mulvey, M. Killen, Child Dev. 86(3), 681-694 (2015) doi: 10.1111/cdev.12317

[9] A. Bhatia, H.K. Sekhon, G. Kaur, Sci. World J. 2014 (2014) doi: 10.1155/2014/159150

[10] M. Zuk, A.M. Stoehr. Sex differences in susceptibility to infection: An evolutionary perspective. In: Sex Hormones and Immunity to Infection (Springer Verlag, 2010), pp. 1-17. doi: 10.1007/978-3-642-02155-8_1

[11] M.J. Corbel, Brucellosis in humans and animals. WHO/CDS/EPR (2006), edited by C. Giefing-Kröll, P. Berger, G. Lepperdinger, B. Grubeck-Loebenstein, Aging Cell. 14, 3 (2015)

[12] F. Guerra-Silveira, F. Abad-Franch, PLoS One 8, e62390 (2013) In: C. Giefing-Kröll, P. Berger, G. Lepperdinger, B. Grubeck-Loebenstein, Aging Cell. 14, 3 (2015) 
[13] G. McCartney, L. Mahmood, A.H. Leyland, G.D. Batty, K. Hunt, Tob. Control 20, 166-168 (2011) In: C. Giefing-Kröll, P. Berger, G. Lepperdinger, B. GrubeckLoebenstein, Aging Cell. 14, 3 (2015)

[14] A. Oksuzyan, K. Juel, J.W. Vaupel, K. Christensen, Aging Clin. Exp. Res. 20, 91-102 (2008) In: C. Giefing-Kröll, P. Berger, G. Lepperdinger, B. GrubeckLoebenstein, Aging Cell. 14, 3 (2015)

[15] J. Vina, C. Borras, J. Gambini, J. Sastre, F.V. Pallardo, Sci. Ageing Knowl. Environ. 2005, pe17. (2005) In: C. Giefing-Kröll, P. Berger, G. Lepperdinger, B. GrubeckLoebenstein, Aging Cell. 14, 3 (2015)

[16] E.N. Fish, Nat. Rev. Immunol. 8, 737-744 (2008) In: C. Giefing-Kröll, P. Berger, G. Lepperdinger, B. Grubeck-Loebenstein, Aging Cell. 14, 3 (2015)

[17] D.P. Robinson, M.E. Lorenzo, W. Jian, S.L. Klein, PLoS Pathog 7(7), e1002149 (2011) doi: 10.1371/journal.ppat.1002149

[18] K. Hayashida, I. Shoji, L. Deng, D. Jiang, Y. Ide, H. Hotta, Microbiol. Immunol. 54(11), 684 (2010)

[19] S.L. Klein, S. Huber, Sex differences in susceptibility to viral infection, edited by S.L. Klein, C.W. Roberts, Sex Hormones and Immunity to Infection (Springer-Verlag, 2010), p. 93-122

[20] M. Rodriguez-Garcia, N. Biswas, M.V. Patel, F.D. Barr, S.G. Crist, C. Ochsenbauer, C.R. Wira, Plos One. 8(4), e62069 (2013)

[21] R.G. Rank, H.J. White, A.J. Hough, J.N. Pasley, A.L. Barron, Infect. Immun. 38(2), 699 (1982)

[22] T. Agrawal, V. Vats, P.K. Wallace, S. Salhan, A. Mittal, RB\&E 6, 46 (2008)

[23] F.O. Dimayuga, J.L. Reed, G.A. Carnero, C. Wang, E.R. Dimayuga, V.M. Dimayuga, A. Perger, M.E. Wilson, J.N. Keller, A.J. Bruce-Keller, J. Neuroimmunol. 161, 123-136 (2015) doi: 10.1016/j.jneuroim.2004.12.016

[24] M. Dumont-Lagacé, C. St-Pierre, C. Perreault, Sci. Rep. 5(12895) 1, 8-9 (2015)

[25] M. Butterworth, B. McClellan, M. Allansmith, Nature. 214, 1224-1225 (1967)

[26] N.J. Olsen, W.J. Kovacs, Endocine Rev. 17, 369 (1996)

[27] M. Zuk, K. McKean, Int. J. Parasitol. 26, 1021 (1996)

[28] X. Li, Y. Xu, L. Ma, L. Sun, G. Fu, Y. Hou, PLoS One. 4(12), e8412, 1 (2009)

[29] R.G. Lahita, H.L. Bradlow, H.G. Kunkel, J. Fishman, J. Clin. Endocrinol. Metab. 53, 174 (1981) In: X. Li, Y. Xu, L. Ma, L. Sun, G. Fu, Y. Hou, PLoS One. 4(2), e8412, 1 (2009)

[30] D.A. Cohn, Clin. Exp. Immunol. 38(2), 218-227 (1979). In: J.I. Morton, D.A. Weyant, B.V. Siegel, B. Golding, Immunol. 44(4), 661, 666-667 (1981)

[31] J.R. Roubinian, N. Talal, J.S. Greenspan, J.R. Goodman, P.K. Siiteri, J. Exp. Med. 147(6), 1568-1583 (1978) In: J.I. Morton, D.A. Weyant, B.V. Siegel, B. Golding, Immunol. 44(4), 661, 666-667 (1981)

[32] A.D. Steinberg, K.A. Melez, E.S. Raveche, J.P. Reeves, W.A. Boegel, P.A. Smathers, J.D. Taurog, L. Weinlein, M. Duvic, Arthritis Rheum. 22(11), 1170-1176 (1979) In: J.I. Morton, D.A. Weyant, B.V. Siegel, B. Golding, Immunol. 44(4), 661, 666-667 (1981)

[33] J.I. Morton, D.A. Weyant, B.V. Siegel, B. Golding, Immunol. 44(4), 661, 666-667 (1981)

[34] M.K. Tripathi, R. Singh, Plos One. 9(8), e104431 (2014)

[35] J. Alexander, W.H. Stimson, Parasit. Today. 4, 189-193 (1988)

[36] S.C. Mills, A. Grapputo, I. Jokinen, E. Koskela, T. Mappes, T. Poikonen, Evolution. 64(1), $172(2010)$ 
[37] L. Merrill, T.E. Stewart, P.L. González-Gómez, A.L. O’Loghlen, J.C. Wingfield, V.A. Ellis, S.I. Rothstein, PBZ. 88(1), 11, 15-16 (2015)

[38] C.G. Greenman, B. Lynn, M. Hau, PBZ. 78(1), 60, 63-64 (2005)

[39] B.J. Prendergast, S.D. Bilbo, R.G. Nelson, J. Neiroendocrinol. 17 (2005)

[40] J.A. Clovis do Prado, A.M.A. Levy, M. Paula Leal, E. Bernard, J. Kardos Kloetzel, Parasitol. Res. 85, 826 (1999) 